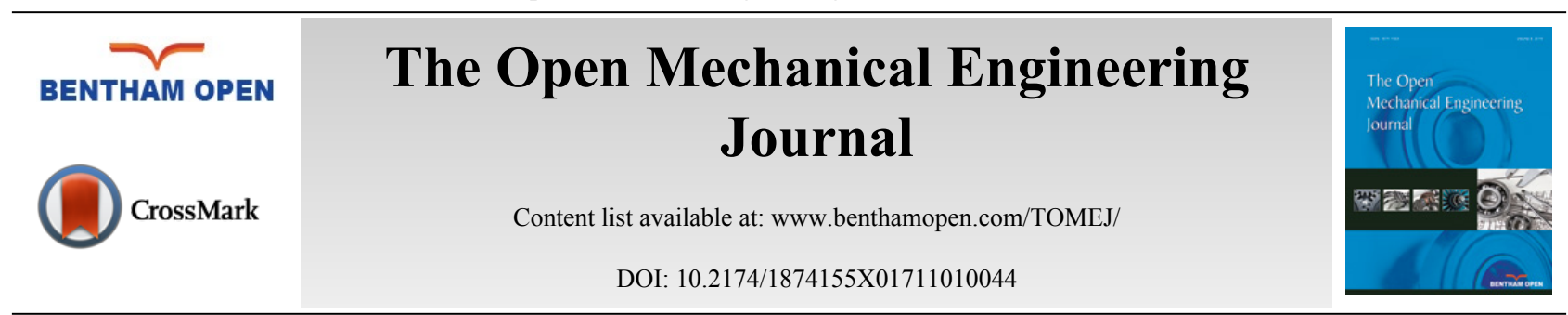

RESEARCH ARTICLE

\title{
Contribution of Bamboo for Vibratory Comfort in Biomechanics of Cycling
}

\author{
Xavier Chiementin ${ }^{1, *}$, Samuel Crequy ${ }^{6}$, Robin Feron ${ }^{2}$, Marcela Munera ${ }^{3}$, Ellie Abdi ${ }^{4}$, Thomas \\ Provot $^{5}$ and Redha Taiar ${ }^{1}$ \\ ${ }^{I}$ GRESPI, Moulin de la Housse, Université de Reims Champagne Ardenne, Reims, France \\ ${ }^{2} I N$ 'BO, ZA Les Bouleaux, Les Voivres, France \\ ${ }^{3}$ Escuela Colombiana de Ingeniería Julio Garavito, Bogotá D.C., Colombia \\ ${ }^{4}$ Montclair State University, Upper Montclair, New Jersey, USA \\ ${ }^{5} E P F, 3$ bis rue Lakanal, Sceaux, France \\ ${ }^{6}$ Arts et Métiers ParisTech, MSMP / EA7350, Châlons-en-Champagne, France
}

Received: June 28, 2017

Revised: August 18, 2017

Accepted: August 21, 2017

\section{Abstract:}

\section{Background:}

Vibrations in cycling produced by road irregularities could cause health problems and affect the cyclist's comfort and performance. Therefore researchers and manufacturers focus their efforts to reduce the vibrations.

\section{Objective:}

The agro materials appear to consume important properties which help in reducing the values of vibrations. This study offers a perspective on the agro materials' contribution in the bicycle design.

\section{Methods:}

Three bicycle frames were compared in two situations: (i) real locomotion conditions at three speeds 15,25 , and $35 \mathrm{~km} / \mathrm{h}$ on slightly grainy road with paved sector and bumps, and (ii) laboratory conditions on a vibrating platform with frequencies ranging between 20 and $80 \mathrm{~Hz}$. The used frames' materials were carbon, aluminum and agro materials (bamboo and flax).

The first protocol measured the effective values in four points of the bicycle (fork, stay, stem, and saddle) in real locomotion condition. The transmissibility was calculated between the input points of vibration and the output points in contact with the rider. The second protocol defined dynamic behavior of the three frames on a vibrating platform at the range of 20-80 Hz.

\section{Results:}

It was noted that the Root Mean Square values (RMS) were significantly higher with the agro materials in $44.4 \%$ of the cases and the values were significantly lower in 1 case (Road with $15 \mathrm{~km} / \mathrm{h}$ ). The agro materials absorbed a significant part of vibrations in comparison to other materials $(19.1 \%, 14.7 \%$, and $17.2 \%$ for agro materials, aluminum, and carbon, respectively).

\section{Conclusion:}

Vibration comfort for cyclists is related to the choice of the frame. The contribution of relevant biomaterials can be relevant. Indeed, agro materials have remarkable properties for the absorption of vibrations.

Keywords: Vibration, Bike comfort, Whole body vibration, Biomechanics.

* Address correspondence to this author at the GRESPI, Moulin de la Housse, Université de Reims Champagne Ardenne, Reims, France; Tel: +33-326918677; E-mail: xavier.chiementin@univ-reims.fr 


\section{INTRODUCTION}

The cycling manufacturers are constantly in search of innovations to design equipment . Some of these innovations are aimed to improve the cyclists' comfort by providing new materials. The agro materials are implemented in many industrial applications such as automotive, food, sustainable energy [1]. Apart from the sustainability, agro materials have stimulating mechanical and energy characteristics like Bamboo which belongs to the family of Gramineae. More specifically Gramineae with the stem woody ones called Poaceae which is flexible and resistant. There are approximately 2300 species of bamboo pertaining to 75 kinds. Concerning its mechanical properties $[2,3]$ the axial tensile Young's modulus fluctuates from 5 to $25 \mathrm{GPa}$, and the axial tensile strength differs from 100 to $800 \mathrm{MPa}$, for specimens taken from the inner and outer culm. Other authors $[4,5]$ showed that compressive strength increases with the height of the culm and with low moisture content [4,6]. Some studies found that [5] the compressive strength of Moso bamboo increases with height along the culm from $45 \mathrm{MPa}$ to $65 \mathrm{MPa}$ and [4] the flexural strength increases from $70 \mathrm{MPa}$ in the green state to $103 \mathrm{MPa}$ for air-dried bamboo. In cycling, comfort is partly linked to the phenomena of vibration. This phenomenon is produced by the road irregularities and the intensities of these irregularities can be high levels [7]. Alternatively, this high level can produce an increase of muscle activity in cycling [8] and significantly with the increase of the energy expenditure (oxygen uptake and lactate concentration) and ventilation compared to cycling without vibration with similar power output $[9,10]$. In contrast, diseases are usually associated to hand-arm system [11 - 13] and to the knee ligaments and meniscus, lower back and shoulder [14, 15]. Thus, numerous studies have analysed the dynamic behavior of the bicycle to limit or to control the vibratory transfer. (For the assessment of this dynamic response, depending on the type of bicycle used, different studies have taken into account $[16,17]$ its structural characteristics [18, 19] surface roughness [16, 18], speed [16], frequencies of the vibration exposure [7] and its amplitude [20]).

One of the dominant factors in vibration transfer is the frame and this research is based on the frame study. Indeed [21], it has been studied by factor analysis that the influence of the components on vibration transfer in the hand-arm system is favored by the fork and tires while the whole body vibration is mainly due to the wheels and frame. Thus the frame can be studied (i) according to its geometry [22, 23], or (ii) the material [24 - 27]. In relations to geometry [20], a model is developed to estimate the dose of vibration to the hand-arm system. It was shown that the vibration dose can be divided by reducing the distance between the two tires of $1 \mathrm{~cm}$.

The focus of this article is on materials and particularly on bamboo and flax fiber. Bamboo can be considered as a unidirectional continuous fiber with reinforced composite; the distribution of its fibers across thickness of the material is gradient [28]. Damping of bamboo was studied in 1997 [2]. This study showed that the viscoelastic damping behavior of raw bamboo has larger damping coefficient in the course of torsion than during bending. A study [29] analysed the modal properties, including damping of bamboo beam. The study showed three resonance frequencies associated with loss factor in the band $0-400 \mathrm{~Hz}$. The values depend largely on the specimen of bamboo. The application of bamboo damping properties could be noteworthy in cycling. It is worth mentioning that these studies must be completed with other research in real conditions. This paper compares the contribution of this agro material coupled with flax fiber for the design of a bicycle frame on three road profiles. This frame is compared with an aluminium and a carbon frames used commercially.

\section{MATERIALS AND METHODS}

\subsection{Subject}

One healthy trained male cyclist $(1 \mathrm{~m} 73,70 \mathrm{~kg})$ was volunteered to participate in the study, which was approved by the local university ethics review board in agreement with the declaration of Helsinki. The participant was aware of the purpose of the study, and a written informed consent was provided. The exclusion criteria included a history of back pain, acute inflammation in the pelvis and/or lower extremities, acute thrombosis, recent fractures, recent implants, gallstones, kidney or bladder stones, any disease of the spine, peripheral vascular disease, and severe delayed onset of muscle soreness in leg muscles was evident.

\subsection{Material}

Three frames including aluminium, carbon and agro-material were tested Fig. (1). The frame in agro material was made of bamboo to the upper tubes, oblique seat and the stays. A flax fiber and epoxy resin $56 \%$ bio-sourced was used for connections between each bamboo beam. The components which equipped the bicycle frame were identical for each 
configuration of the study. To ensure identical clamping the assembly was carried out with a torque wrench. The characteristics of the frames and peripheral devices are listed in Table $\mathbf{1}$.

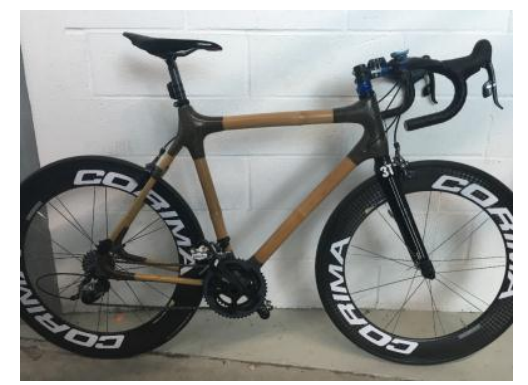

(a)

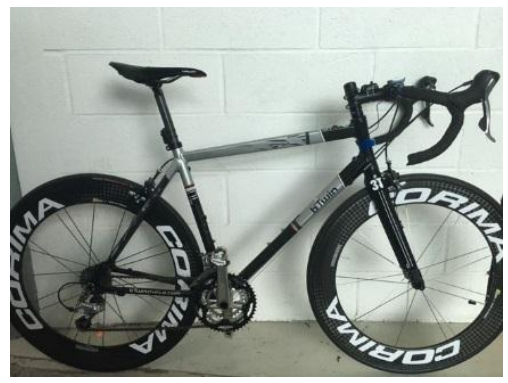

(b)

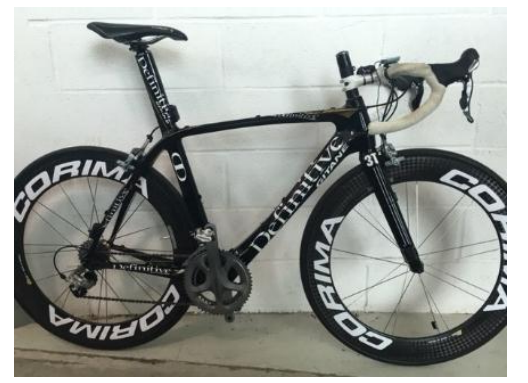

(c)

Fig. (1). Tested bicycles: (a) Agro-material frame, (b) Aluminium frame, (c) Carbon frame.

Table 1. Bike frames and components.

\begin{tabular}{|c|c|c|c|}
\hline Frame & Agro Material & Aluminium & Carbone \\
\hline Weight $(\mathrm{kg})$ & 9.0 & 8.5 & 7.9 \\
\hline Wheels & \multicolumn{2}{|c|}{ Corima carbon, tubeless tire MCC S+ } \\
\hline Tyres & \multicolumn{2}{|c|}{ Continental 23mm } \\
\hline Seat & \multicolumn{2}{|c|}{ San marco Aspide carbone FX team open } \\
\hline Fork & \multicolumn{2}{|c|}{} \\
\hline
\end{tabular}

Four IMU (Inertial Mouvement Unit) HIKOB Fox (Villeurbanne, France) validated in the study of [30] were fixed in the bicycle. Two sensors were on the bottom of the fork and the stays, which were considered as inputs. Two sensors were on the stem and over the seat considered as outputs due to the point of contact with the human body Fig. (2). In the second protocol which was presented in the section 2.3, a fifth IMU sensor was set in the middle of the vibratory plate. The sampling rate was chosen at $1344 \mathrm{~Hz}$ with an amplitude range of $+/-16 \mathrm{~g}$.

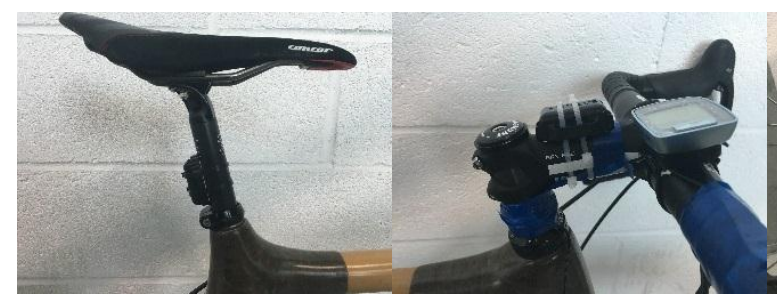

(a) (b)

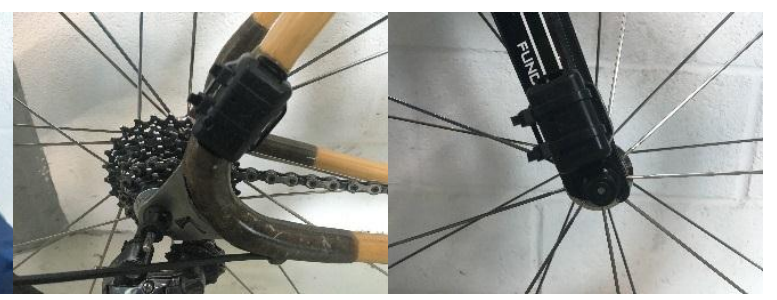

(c) (d)

Fig. (2). Positon of IMU Sensor on (a) seat, (b) stem, (c) stay and (d) fork.

\subsection{Protocols}

The study was composed of two protocols. The first protocol was to assess the effective values in real conditions on 3 types of sectors: slightly rough, paved and with speed bump Fig. (3). These sectors were flat. Twenty measures were performed on each sector at the speeds of 15,25 and $35 \mathrm{~km} / \mathrm{h}$. Rest of 1 minute was allowed in between each try. Speed was visually controlled by the rider on a counter (Sigma BC 16.12, Decathlon, France). The second protocol analysis was to determine the vibration response of the bike on a vibratory plate in the laboratory. Rear and front wheels were positioned sucessively on the middle of the vibratory plate. The plate had a vertical oscillation amplitude of $2 \mathrm{~mm}(600$ FITVIB, Germany). It was controlled by an acquisition system OROS OR35 (OROS, Grenoble, France) to perform a swept sinus in the range of $20-80 \mathrm{~Hz}$ with steps at the range of $2 \mathrm{~Hz}$. 


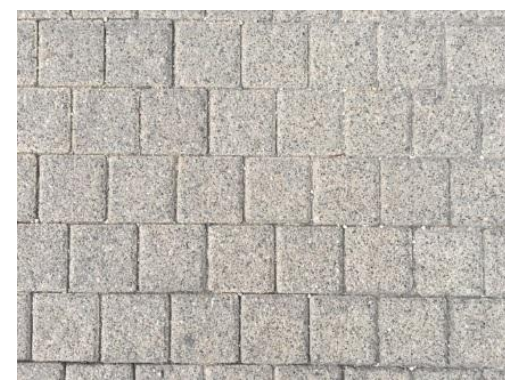

(a)

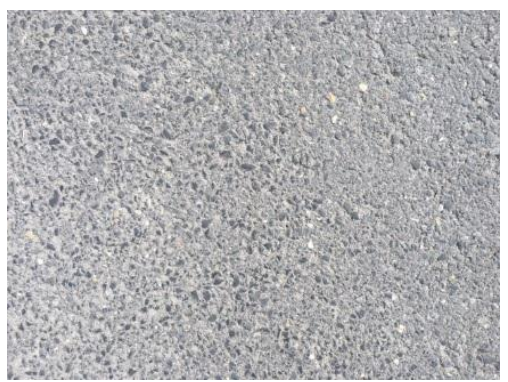

(b)

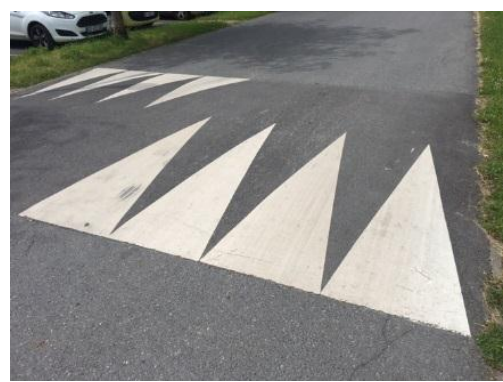

(c)

Fig. (3). Road sectors: (a) Paved, (b) slightly rough and (c) Speed bump.

For protocol 1, the signals were collected during $5 \mathrm{~s}, 3 \mathrm{~s}$, and $2 \mathrm{~s}$ for speeds of 15,25 , and $35 \mathrm{~km} / \mathrm{h}$, respectively, which corresponded to 6720,4032 and 2688 samples and with the same distance of $21 \mathrm{~m}$. This distance included each evaluated sector. The hypothesis was made that the directions of the IMU sensors were similar during the measurements. . Thus, the average values of $\bar{x}, \bar{y}, \bar{z}$, were subtracted from the recorded signal $\mathrm{x}(\mathrm{n}), \mathrm{y}(\mathrm{n}), \mathrm{z}(\mathrm{n})$, for each axis because it reflected the gravity. Afterward, the two parameters were calculated: the total root mean squared (RMS) for each measurement points, shown in equation (1), and the transmissibility coefficient $\mathrm{T}_{\mathrm{ij}}$, shown in equation (2). The Tij was calculated as the ratio of the RMS value with the seat or stem and the fork or stay. Following the Shapiro Wilk test of normality in frequentist statistics, significant differences between the frames were evaluated by Student's t-tests. The statistically significant level was selected to $0.05(\mathrm{p}<0.05)$. Results are presented as mean \pm standard deviation.

$$
\begin{aligned}
& R M S=\sum \frac{(x(n)-x)^{2}}{N}+\sum \frac{(y(n)-\bar{y})^{2}}{N}+\sum \frac{(z(n)-z)^{2}}{N} \\
& T_{i j}=\frac{R M S_{i}}{R M S_{j}}, i: \text { seat or stem, } j: \text { fork or stay }
\end{aligned}
$$

For Protocol 2, the signals were collected during $5 \mathrm{~s}$ within a samplig rate of $2560 \mathrm{~Hz}$ during a stationary condition of the plate. The average value was subtracted from the recorded signal for each axis $\mathrm{x}, \mathrm{y}, \mathrm{z}$ since it reflected the gravity. Next, the RMS values were computed for all IMU positions, equation (1). Lastly, for each excitatory frequency f, the ratio between RMS value of the wheels and the 4 measurement points were calculated. The transmissibility functions were deduced in the range $20-80 \mathrm{~Hz}, \mathrm{~T}_{\mathrm{lab}, \mathrm{jij}}(\mathrm{f})$, (equation 3 ). These functions are dependent on the frequency $\mathrm{f}$ generated by the vibration platform. An amplification of the input was obtained for transmissibility values above 1 . After testing the normality of the data by the test of Shapiro Wilk, significant differences between the frames were evaluated by Student's t-tests. The statistically significant level was selected to $0.05(\mathrm{p}<0.05)$. Results are presented as mean \pm standard deviation.

$$
T_{l a b, i j}(f)=\frac{R M S_{i}(f)}{R M S_{j}(f)}, i: \text { seat or stem, } j: \text { fork or stay }
$$

\section{RESULTS}

\subsection{Real Conditions}

The RMS values which were measured at the four points of measurement are listed in Table 2. The results show a significant difference between the 3 road profiles and between the 3 speeds. The values of $95 \%$ increased in between 15 and $25 \mathrm{~km} / \mathrm{h}$ and values of $56.9 \%$ increased in between 25 and $35 \mathrm{~km} / \mathrm{h}$. The increase in the RMS values by $174 \%$ between the speed bump and the slightly rough road and the $359 \%$ in between paved road and the slightly rough road were evident. In $38.9 \%$ of cases, there was a significant difference in between the aluminium frame and the agromaterial frame, and in $50 \%$ of cases, there was a difference between the carbon frame and agro-material frame. In the case of "slightly rough road to $15 \mathrm{~km} / \mathrm{h}$ ", $1.4 \%$ had a significant difference in favour of the agro. It was noted that the significant differences were $41.7 \%, 54.2 \%$ and $37.5 \%$ at speeds of 15,25 and $35 \mathrm{k} / \mathrm{h}$, respectively. The significant differences in the stem, seat, fork, and stay were $33.3 \%, 55.6 \%, 27.8 \%$ and $61.1 \%$. 
Table 2. RMS values, in $\mathrm{m} / \mathrm{s}^{2}$, measured on stem, seat, stay and fork. The $\mathrm{p}$-value indicates the significance of the test between aluminium or carbon frame and agro material frame.

\begin{tabular}{|c|c|c|c|c|c|c|c|c|c|}
\hline \multirow[b]{2}{*}{ STEM } & \multirow[b]{2}{*}{ Sectors } & \multicolumn{2}{|c|}{ Agro-Material } & \multicolumn{3}{|c|}{ Aluminium } & \multicolumn{3}{|c|}{ Carbon } \\
\hline & & $\mathrm{m}$ & std & $\mathrm{m}$ & std & p-value & $\mathrm{m}$ & std & p-value \\
\hline \multirow[t]{3}{*}{$15 \mathrm{~km} / \mathrm{h}$} & Road & 2.13 & 0.20 & 2.26 & 0.17 & 0.03 & 2.05 & 0.13 & 0.15 \\
\hline & Cobblestone & 9.00 & 0.84 & 8.96 & 0.75 & 0.92 & 8.47 & 0.69 & 0.14 \\
\hline & Speed bump & 5.14 & 0.49 & 4.95 & 0.39 & 0.19 & 4.73 & 0.43 & 0.01 \\
\hline \multirow[t]{3}{*}{$25 \mathrm{~km} / \mathrm{h}$} & Road & 3.26 & 0.26 & 3.20 & 0.30 & 0.52 & 3.17 & 0.36 & 0.35 \\
\hline & Cobblestone & 19.51 & 1.74 & 17.65 & 1.19 & 0.01 & 17.33 & 1.27 & 0.00 \\
\hline & Speed bump & 10.93 & 0.91 & 10.24 & 1.19 & 0.05 & 10.38 & 0.91 & 0.06 \\
\hline \multirow[t]{3}{*}{$35 \mathrm{~km} / \mathrm{h}$} & Road & 5.28 & 0.54 & 5.17 & 0.48 & 0.52 & 4.92 & 0.52 & 0.04 \\
\hline & Cobblestone & 27.41 & 1.7 & 27.17 & 2.38 & 0.80 & 26.23 & 2.03 & 0.16 \\
\hline & Speed bump & 18.23 & 0.87 & 17.74 & 1.75 & 0.27 & 18.04 & 2.41 & 0.75 \\
\hline SEAT & Sectors & \multicolumn{2}{|c|}{ Agro-Material } & \multicolumn{3}{|c|}{ Aluminium } & \multicolumn{3}{|c|}{ Carbon } \\
\hline \multirow[t]{3}{*}{$15 \mathrm{~km} / \mathrm{h}$} & Road & 1.93 & 0.18 & 1.90 & 0.14 & 0.52 & 1.97 & 0.12 & 0.41 \\
\hline & Cobblestone & 7.57 & 0.58 & 7.41 & 0.51 & 0.51 & 7.08 & 0.43 & 0.04 \\
\hline & Speed bump & 4.70 & 0.69 & 4.36 & 0.57 & 0.10 & 4.27 & 0.58 & 0.04 \\
\hline \multirow[t]{3}{*}{$25 \mathrm{~km} / \mathrm{h}$} & Road & 2.88 & 0.23 & 2.73 & 0.24 & 0.05 & 2.95 & 0.33 & 0.40 \\
\hline & Cobblestone & 16.22 & 0.8 & 15.01 & 0.98 & 0.01 & 14.93 & 1.04 & 0.01 \\
\hline & Speed bump & 10.25 & 0.84 & 9.24 & 1.21 & 0.00 & 9.56 & 1.11 & 0.03 \\
\hline \multirow[t]{3}{*}{$35 \mathrm{~km} / \mathrm{h}$} & Road & 4.85 & 0.45 & 4.79 & 0.36 & 0.66 & 4.80 & 0.46 & 0.73 \\
\hline & Cobblestone & 24.90 & 0.3 & 24.59 & 1.52 & 0.59 & 23.44 & 1.24 & 0.01 \\
\hline & Speed bump & 17.63 & 1.56 & 16.33 & 1.91 & 0.02 & 16.45 & 1.89 & 0.04 \\
\hline FORK & Sectors & \multicolumn{2}{|c|}{ Agro-Material } & \multicolumn{3}{|c|}{ Aluminium } & \multicolumn{3}{|c|}{ Carbon } \\
\hline \multirow[t]{3}{*}{$15 \mathrm{~km} / \mathrm{h}$} & Road & 2.81 & 0.28 & 2.91 & 0.21 & 0.22 & 2.84 & 0.17 & 0.72 \\
\hline & Cobblestone & 11.09 & 0.85 & 10.74 & 0.87 & 0.37 & 10.08 & 0.75 & 0.01 \\
\hline & Speed bump & 5.97 & 1.16 & 5.55 & 0.29 & 0.13 & 5.60 & 0.39 & 0.19 \\
\hline \multirow[t]{3}{*}{$25 \mathrm{~km} / \mathrm{h}$} & Road & 4.41 & 0.36 & 4.20 & 0.39 & 0.09 & 4.30 & 0.46 & 0.40 \\
\hline & Cobblestone & 28.41 & 2.76 & 24.77 & 2.64 & 0.01 & 21.58 & 1.75 & 0.00 \\
\hline & Speed bump & 11.66 & 1.09 & 11.07 & 1.30 & 0.13 & 11.30 & 0.77 & 0.23 \\
\hline \multirow[t]{3}{*}{$35 \mathrm{~km} / \mathrm{h}$} & Road & 6.77 & 0.79 & 6.66 & 0.60 & 0.61 & 6.12 & 0.64 & 0.01 \\
\hline & Cobblestone & 37.08 & 1.91 & 36.00 & 3.18 & 0.37 & 29.96 & 1.53 & 0.00 \\
\hline & Speed bump & 20.00 & 1.11 & 19.51 & 1.79 & 0.30 & 20.01 & 1.97 & 0.99 \\
\hline STAY & Sectors & \multicolumn{2}{|c|}{ Agro-Material } & \multicolumn{3}{|c|}{ Aluminium } & \multicolumn{3}{|c|}{ Carbon } \\
\hline \multirow[t]{3}{*}{$15 \mathrm{~km} / \mathrm{h}$} & Road & 3.01 & 0.23 & 2.33 & 0.15 & 0.00 & 2.52 & 0.13 & 0.00 \\
\hline & Cobblestone & 8.33 & 0.51 & 7.94 & 0.46 & 0.09 & 7.90 & 0.36 & 0.04 \\
\hline & Speed bump & 5.66 & 0.65 & 4.89 & 0.58 & 0.00 & 4.99 & 0.69 & 0.00 \\
\hline $25 \mathrm{~km} / \mathrm{h}$ & Road & 4.75 & 0.33 & 3.51 & 0.26 & 0.00 & 3.96 & 0.35 & 0.00 \\
\hline & Cobblestone & 16.54 & 0.72 & 15.84 & 1.19 & 0.13 & 16.79 & 1.33 & 0.61 \\
\hline & Speed bump & 11.42 & 0.69 & 10.19 & 1.17 & 0.00 & 11.22 & 1.22 & 0.53 \\
\hline $35 \mathrm{~km} / \mathrm{h}$ & Road & 6.98 & 0.49 & 5.76 & 0.37 & 0.00 & 6.24 & 0.52 & 0.00 \\
\hline & Cobblestone & 26.72 & 1.19 & 26.66 & 2.01 & 0.94 & 26.33 & 1.59 & 0.55 \\
\hline & Speed bump & 19.64 & 1.63 & 18.20 & 1.80 & 0.01 & 19.33 & 1.89 & 0.59 \\
\hline
\end{tabular}

The transmissibilities values are given in Table 3. The values are inferior to 1 which shows that frames did not amplify the vibratory level recorded at the bottom of the fork and the stay. Mean transmissibilities were $80.9 \%, 85.3 \%$ and $82.8 \%$ for frames in agro materials, aluminum and carbon, respectively. The table demonstrates that $37.5 \%$ of cases have a significant difference between aluminum or carbon frames and agro materials frame for the benefit of agro materials. In particular, the transmissibility between the stay and the seat or the stem for the agro materials frame was significantly lower than the aluminum frame regardless of road and speed conditions and it was significantly lower than the carbon frame for speed $15 \mathrm{~km} / \mathrm{h}$. Transmissibilities between the fork and the seat or stem were not significantly different except for the carbon at $35 \mathrm{~km} / \mathrm{h}$. 
Table 3. Transmissibility coefficients.

\begin{tabular}{|c|c|c|c|c|c|c|c|c|}
\hline \multirow[t]{2}{*}{ Fork - Stem } & \multicolumn{2}{|c|}{ Agro-Material } & \multicolumn{3}{|c|}{ Aluminium } & \multicolumn{3}{|c|}{ Carbon } \\
\hline & $\mathrm{m}$ & std & $\mathrm{m}$ & std & p-value & $\mathrm{m}$ & std & p-value \\
\hline $15 \mathrm{~km} / \mathrm{h}$ & 0,82 & 0,08 & 0,83 & 0,06 & 0,18 & 0,79 & 0,07 & 0,17 \\
\hline $25 \mathrm{~km} / \mathrm{h}$ & 0,81 & 0,11 & 0,82 & 0,09 & 0,64 & 0,82 & 0,09 & 0,49 \\
\hline $35 \mathrm{~km} / \mathrm{h}$ & 0,83 & 0,09 & 0,83 & 0,08 & 1,00 & 0,86 & 0,07 & 0,04 \\
\hline \multirow[t]{2}{*}{ Stay- Stem } & \multicolumn{2}{|c|}{ Agro-Material } & \multicolumn{3}{|c|}{ Aluminium } & \multicolumn{3}{|c|}{ Carbon } \\
\hline & $\mathrm{m}$ & std & $\mathrm{m}$ & std & p-value & $\mathrm{m}$ & std & p-value \\
\hline $15 \mathrm{~km} / \mathrm{h}$ & 0,86 & 0,15 & 1,02 & 0,08 & 0,00 & 0,92 & 0,12 & 0,03 \\
\hline $25 \mathrm{~km} / \mathrm{h}$ & 0,89 & 0,19 & 0,99 & 0,08 & 0,00 & 0,90 & 0,10 & 0,89 \\
\hline $35 \mathrm{~km} / \mathrm{h}$ & 0,88 & 0,12 & 0,95 & 0,08 & 0,00 & 0,89 & 0,11 & 0,74 \\
\hline \multirow[t]{2}{*}{ Fork - Seat } & \multicolumn{2}{|c|}{ Agro-Material } & \multicolumn{3}{|c|}{ Aluminium } & \multicolumn{3}{|c|}{ Carbon } \\
\hline & $\mathrm{m}$ & std & $\mathrm{m}$ & std & p-value & $\mathrm{m}$ & std & p-value \\
\hline $15 \mathrm{~km} / \mathrm{h}$ & 0,73 & 0,09 & 0,71 & 0,08 & 0,31 & 0,72 & 0,07 & 0,66 \\
\hline $25 \mathrm{~km} / \mathrm{h}$ & 0,73 & 0,13 & 0,72 & 0,10 & 0,59 & 0,75 & 0,09 & 0,30 \\
\hline $35 \mathrm{~km} / \mathrm{h}$ & 0,78 & 0,11 & 0,76 & 0,09 & 0,46 & 0,80 & 0,06 & 0,15 \\
\hline \multirow[t]{2}{*}{ Stay- Seat } & \multicolumn{2}{|c|}{ Agro-Material } & \multicolumn{3}{|c|}{ Aluminium } & \multicolumn{3}{|c|}{ Carbon } \\
\hline & $\mathrm{m}$ & std & $\mathrm{m}$ & std & p-value & $\mathrm{m}$ & std & $\mathrm{p}$-value \\
\hline $15 \mathrm{~km} / \mathrm{h}$ & 0,77 & 0,11 & 0,87 & 0,05 & 0,00 & 0,83 & 0,05 & 0,00 \\
\hline $25 \mathrm{~km} / \mathrm{h}$ & 0,80 & 0,16 & 0,86 & 0,07 & 0,01 & 0,82 & 0,07 & 0,43 \\
\hline $35 \mathrm{~km} / \mathrm{h}$ & 0,82 & 0,11 & 0,88 & 0,04 & 0,00 & 0,83 & 0,05 & 0,90 \\
\hline
\end{tabular}

\subsection{Laboratory Conditions}

Prior to the calculation of the transmibility functions, the frequency ranges of the vibratory plate compared to road profile were validated. Fig. (4) shows the time signal to 3 seconds and its spectrum on the range $0-200 \mathrm{~Hz}$ for the three field conditions. For this exemple, $95 \%$ of the spectrum was in the $15-72 \mathrm{~Hz}$ range. There was a structural resonance on $120-180 \mathrm{~Hz}$ amplitude range less than $0.1 \mathrm{~m} / \mathrm{s}$.

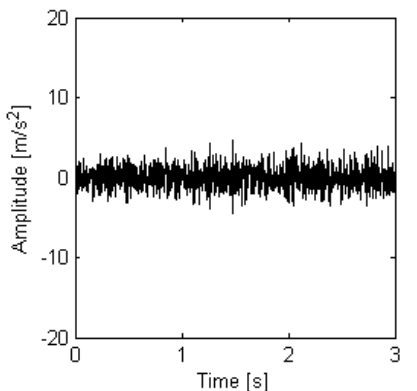

(a)

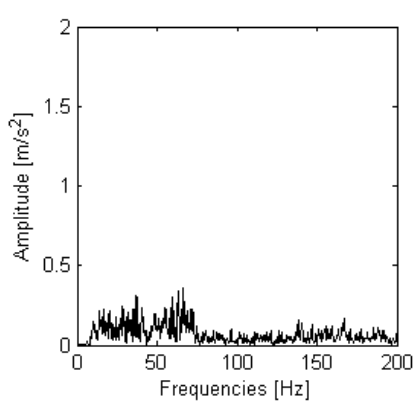

(d)

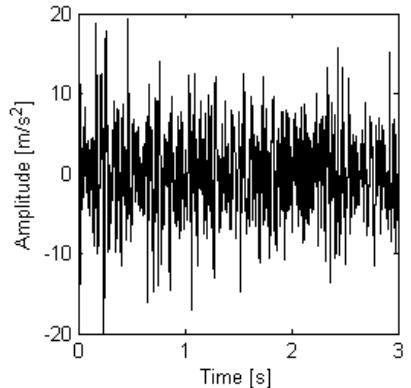

(b)

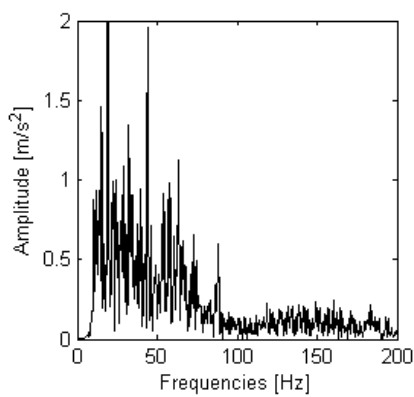

(e)

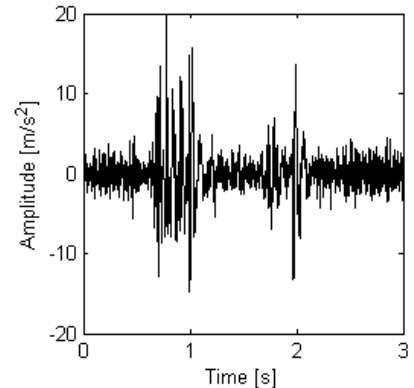

(c)

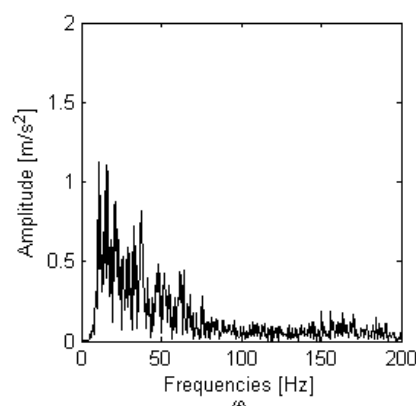

(f)

Fig. (4). Example of time signal $(a, b, c)$ and its spectra $(d, e, f)$ on slighty rough road $(a, d)$ on paved sector (b,e) with speed bump (c,f) at $25 \mathrm{~km} / \mathrm{h}$ for the agro material bike. 
An analysis of the median frequency in Table 4 indicates that the frequency values of the requested range decreased with the speed for the three frame. On the slightly rough road, the median frequencies were $65.5,41.4$, and $38.3 \mathrm{~Hz}$ for speeds of 15,25 and $35 \mathrm{~km} / \mathrm{h}$, respectively. On the paved sector, the median frequencies were $32.3,36.1$, and $39.5 \mathrm{~Hz}$ for speeds 15,25 and $35 \mathrm{~km} / \mathrm{h}$, respectively. The speed bump showed median frequencies of $27.5,28.2$, and $28.5 \mathrm{~Hz}$ for speeds 15,25 and $35 \mathrm{~km} / \mathrm{h}$, respectively. These results justify the use of the vibratory plate ranging fromrange $20-80 \mathrm{~Hz}$ representing $92 \%$ of the excited range on the field.

Table 4. Median frequencies $(\mathrm{Hz})$.

\begin{tabular}{|c|c|c|c|c|c|c|c|}
\hline Speed & Sectors & \multicolumn{2}{|c|}{ Agro Material } & \multicolumn{2}{|c|}{ Aluminium } & \multicolumn{2}{c|}{ Carbon } \\
\hline & & $\mathbf{m}$ & $\mathbf{s t d}$ & $\mathbf{m}$ & $\mathbf{s t d}$ & $\mathbf{m}$ & std \\
\hline $15 \mathrm{~km} / \mathrm{h}$ & Road & 63.0 & 6.4 & 67.6 & 3.9 & 65.8 & 3.2 \\
\hline & Cobblestone & 31.2 & 4.0 & 32.5 & 2.24 & 33.1 & 3.4 \\
\hline & Speed bump & 24.9 & 4.1 & 27.8 & 4.9 & 29.8 & 6.44 \\
\hline $25 \mathrm{~km} / \mathrm{h}$ & Route & 40.8 & 2.7 & 41.3 & 3.5 & 42.1 & 4.8 \\
\hline & Cobblestone & 37.5 & 0.7 & 36.4 & 0.8 & 34.3 & 1.7 \\
\hline & Speed bump & 29.0 & 4.2 & 27.9 & 3.5 & 27.7 & 3.1 \\
\hline $35 \mathrm{~km} / \mathrm{h}$ & Road & 37.6 & 3.5 & 38.6 & 3.6 & 38.7 & 4.5 \\
\hline & Cobblestone & 40.8 & 1.5 & 40.0 & 2.9 & 37.8 & 2.6 \\
\hline & Speed bump & 29.5 & 3.4 & 27.8 & 3.1 & 28.1 & 3.5 \\
\hline
\end{tabular}

The transmissibility functions between the rear and the front wheels and the four measurement points are listed respectively in Figs. (5 and 6), respectively. An amplification of the input signal on a certain frequency range is highlighed which shows that the ranges are having a transmissibility superior to 1 . Those ranges of amplification were $32-52 \mathrm{~Hz}$ at the fork, $40-50 \mathrm{~Hz}$ at the stem, $35-50 \mathrm{~Hz}$ at the stay and $40-47 \mathrm{~Hz}$ at the seat. There was a further range in between $55-65 \mathrm{~Hz}$ for aluminum at the fork. Aimed at the sollicitation on the front wheel, the amplification was more important for agro material frame than the other two frames intending a wide frequency range. Nevertheless in the range of $55-65 \mathrm{~Hz}$ it has similar characteristics to the carbon while the aluminum frame amplified the exposure level. For a sollicitation on the rear wheel, the 3 frames had similar transmissibilities behaviors over the range studied.

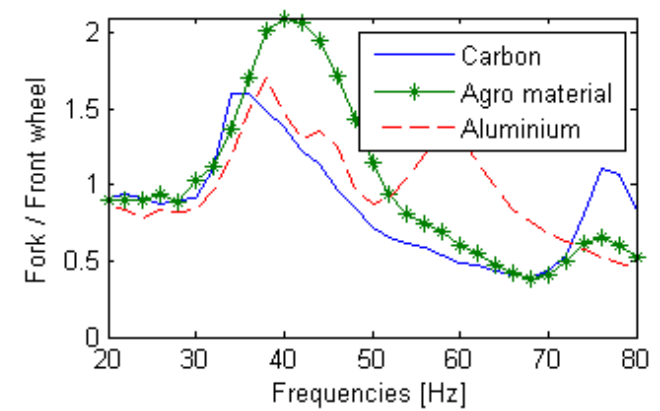

(a)

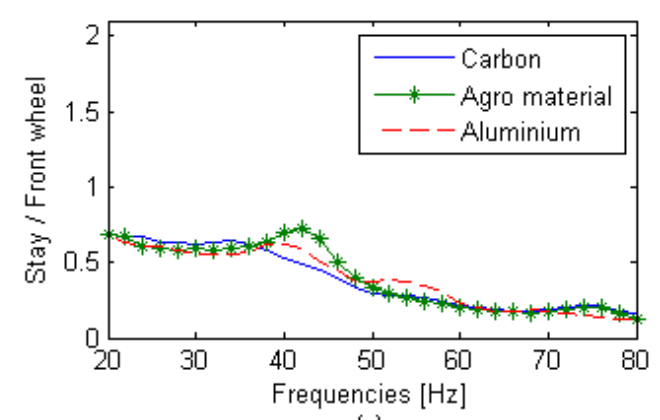

(c)

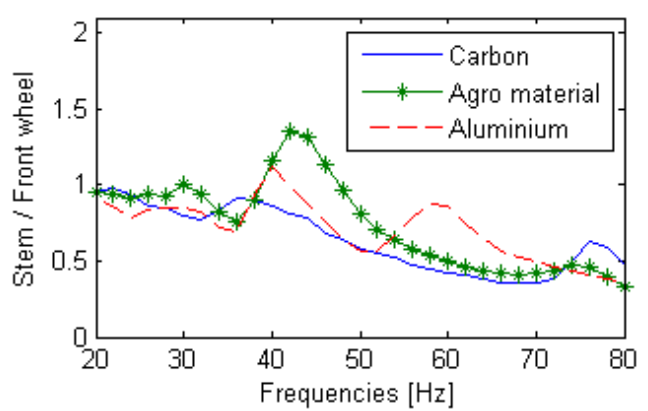

(b)

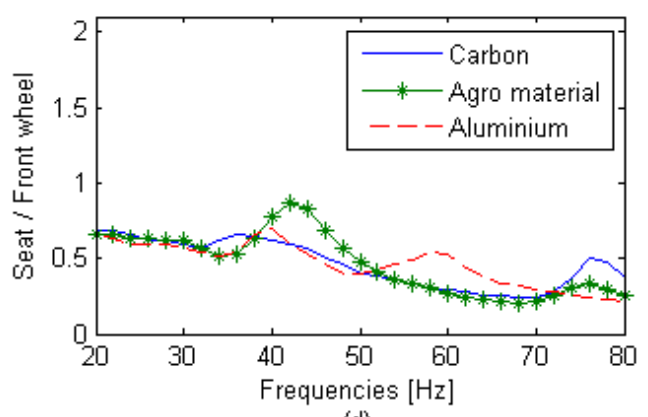

(d)

Fig. (5). Transmissibility function between front wheel and (a) fork, (b) stem, (c) stay and (d) seat. 


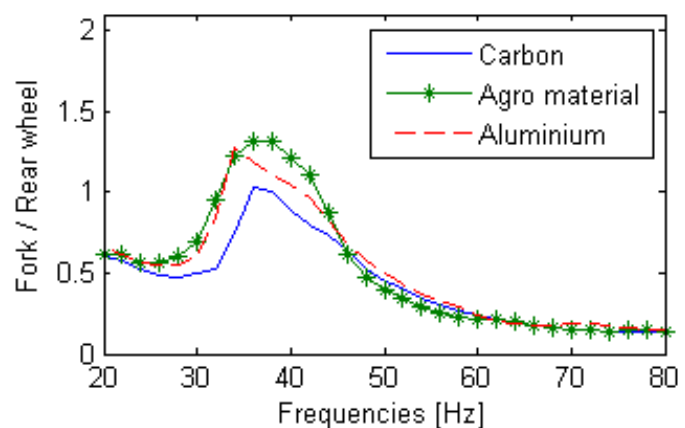

(a)

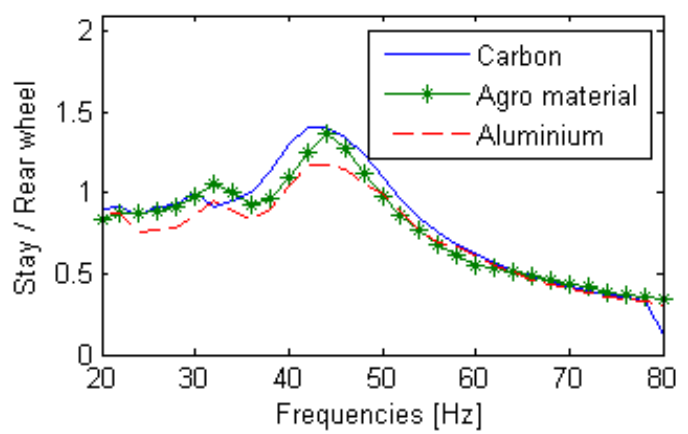

(c)

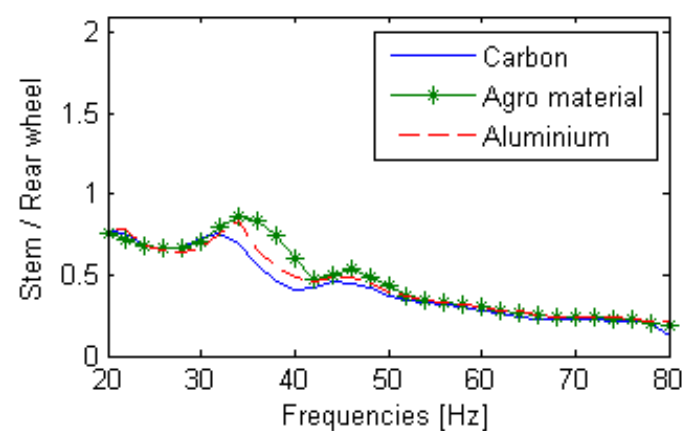

(b)

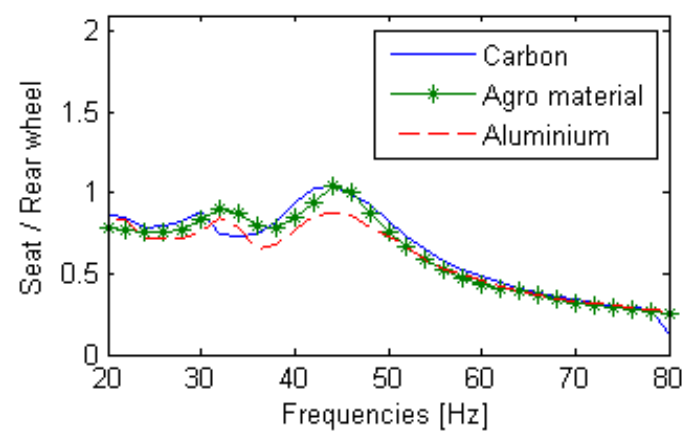

(d)

Fig. (6). Transmissibility function between rear wheel and (a) fork, (b) stem, (c) stay and (d) seat.

\section{DISCUSSION}

As shown by numerous studies $[16,18]$ the exposure level is different on the three road profiles with respect to high values on the pavement profile. The speed also has an impact on vibratory levels recorded. The level is increased between 56.9 and $95 \%$.

The analysis of the RMS values allowed to compare the three bike frames and showed that the values were lower for carbon. The agro material frame had the higher results with one single condition of comfort gain $(15 \mathrm{~km} / \mathrm{h}$ on slighty rough road compared with the aluminum frame). This fact was related to the solicitation of median frequency of $65.5 \mathrm{~Hz}$ and amplification range of $55-65 \mathrm{~Hz}$ for the aluminum frame highlighted by the laboratory protocol. On a slighy rough profile, the agro material frame had properties which were similar or better than aluminium. In contrast, the agro material frame had low characteristics at the speed of $25 \mathrm{~km} / \mathrm{h}$, which is highlighted by the laboratory protocol and the median frequencies. At this speed, the median frequencies were between 28.2 and $41.4 \mathrm{~Hz}$. This fact coincides with the high amplification factors for the agro material frame (amplification value reaching 2.1 against 1.6 and 1.5 for the other two frames). The agro material frame appeared to remain penalized by the attachment between the wheel and the stay. The vibration level was excessive compared to other frame which affected the vibratory level received at the seat. In general the stay had a vibratory level of $14 \%$ higher than carbon or aluminum. This increase was only $7 \%$ in the seat which may explain the effect of the stay itself. Vibration doses were in between $9 \%$ to $18 \%$ higher at the wheels compared to other two frames nonetheless these levels were in between $7 \%$ and $9 \%$ only for seat and stem, respectively. This result indicated that the agro material frame had excellent properties in the transmission of vibrations but it has higher RMS values.

The analysis of the transmissibility confirmed that the agro material frame had a better absorption than the aluminum frame particularly between the stay and seat/stem. This result was in correspondence with another work [31]. In general, it had the same damping properties as a carbon frame; however, showed better results for some speeds. The transmissibilities average values of 0.88 in between the stay and the stem, 0.82 in between the fork and the stem, 0.75 in between the fork and the seat, 0.80 in between the stay and the seat, were obtained. From seat stay, an absorption gain was noted for the agro material frame compared to aluminium frame $(9 \%)$ and compared to carbon frame $(2 \%)$. The transmissibilities of the vibratory stressed from the fork were identical to the three frames. This could be explained 
through the preponderance of the absorption by the fork. The fact is confirmed by a study [21], where it was shown that the fork and the frame were highly pertinent elements in the transmission of vibrations. The transmissibility functions resonanced frequencies range were $30-50 \mathrm{~Hz}$ for the wrist, $16-30 \mathrm{~Hz}$ for the forearm, $20-90 \mathrm{~Hz}$ for the eyeball and $10-12 \mathrm{~Hz}$ for spine [32]. Carbon frames and aluminum were preferred for disease prevention at the wrist, forearm, and visual performance. It was noted that the aluminum frame can affect the eyeball more broadly because of its modal properties superior to $55 \mathrm{~Hz}$. The effect on the spine could not be treated here due to the mechanical limitations of the bench. The bench could not generate frequency under $20 \mathrm{~Hz}$ yet, spectral analysis revealed the vibratory stresses superior to $15 \mathrm{~Hz}$ on field conditions.

\subsection{Recommendation/Perspectives}

Although the agro material frame did not have significantly different vibration characteristics in $55.5 \%$ of cases, this non-optimized frame was very promising. According to the results, it is recommended to modify the design at the connection between the hub and the stay. The junction in front of the frame and in between the fork and the stem can be optimized to compensate the vibratory amplification, however, the level is higher than the other two frames. These changes would affect the general behavior of the frame. The optimization involved the natural geometry of bamboo. Currently there is a need for empirical and numerical studies which could bring more information including the selection of thicknesses, the position of the nodes, the length of the internodes, and the position of the reinforcement with flax fiber. The contribution of the modal analysis could help to understand the natural deformation of the frame in particular, medio-lateral plane.

This study is subject to two bias. First bias indicates that the bikes' masses were different at 5.6\% and $12.2 \%$ less for aluminum and carbon frames, respectively. However, these differences cannot explain the dissimilarities in doses only because according to Newton's second law, acceleration increases with decreasing mass for identical stress forces. The second bias is related to the repeatability of the agro material production frame. Are the results similar between two identical agro material frames knowing that production is handmade?

\section{CONCLUSION}

Vibration comfort for cyclists is related to the choice of the frame. The contribution of relevant biomaterials could be relevant. Interestingly enough agro materials have the ability to absorp vibrations. This paper focused on the study of bamboo use and flax fiber to design a road bike frame. The study was achieved by comparing the dynamical behavior of three frames on 3 road profiles: slightly rough, speed bump and cobblestones. The comparison with aluminum frame and carbon frame showed an absorption rate higher for agro materials ( $9 \%$ compared to aluminum and $2 \%$ compared to carbon), Nevertheless the tested bike had higher vibration dose level on the inputs (fork and stay). This fact showed that the design should be redefined. The contribution of agros materials reduces the transfer function yet the frame should be optimized, and in particular weight needs to be decreased.

\section{CONSENT FOR PUBLICATION}

Not applicable.

\section{CONFLICT OF INTEREST}

The authors declare no conflict of interest, financial or otherwise.

\section{ACKNOWLEDGEMENTS}

Declared none.

\section{REFERENCES}

[1] A. Franscisco, R. Lahr, H.S. Junior, and J. Fiorelli, Non-conventional Building Materials based on agro-industrial wastes., Tiliform: Bauru, SP, 2015.

[2] S. Amada, and R. Lakes, "Viscoelastic properties of bamboo", J. Mater. Sci., vol. 32, no. 10, pp. 2693-2697, 1997. [http://dx.doi.org/10.1023/A:1018683308211]

[3] Z.P. Shao, C.H. Fang, S.X. Huang, and G.L. Tian, "Tensile properties of moso bamboo (phyllostachys pubescens) and its components with respect to its fiber-reinforced composite structure", Wood Sci. Technol., vol. 44, pp. 655-666, 2010. [http://dx.doi.org/10.1007/s00226-009-0290-1] 
[4] A.W. Lee, X. Bai, and P.N. Peralta, "Selected physical and mechanical properties of giant timber bamboo grown in South Carolina", For. Prod. J., vol. 44, pp. 40-46, 1994.

[5] T.Y. Lo, H. Cui, and H. Leung, "The effect of fiber density on strength capacity of bamboo", Mater. Lett., vol. 58, no. 21, pp. 2595-2598, 2004.

[http://dx.doi.org/10.1016/j.matlet.2004.03.029]

[6] K.F. Chung, and W.K. Yu, "Mechanical properties of structural bamboo for bamboo scaffoldings", Eng. Struct., vol. 24, pp. 429-442, 2002. [http://dx.doi.org/10.1016/S0141-0296(01)00110-9]

[7] X. Chiementin, M. Rigaut, S. Crequy, and W. Bertucci, "Hand-arm vibration in cycling", J. Vib. Control, vol. 19, no. 16, pp. 2551-2560, 2013. [http://dx.doi.org/10.1177/1077546312461024]

[8] P. Arpinar-Avsar, G. Birlik, Ö.C. Sezgin, and A.R. Soylu, "The effects of surface-induced loads on forearm muscle activity during steering a bicycle", J. Sports Sci. Med., vol. 12, no. 3, pp. 512-520, 2013. [PMID: 24149159]

[9] D. Filingeri, M. Jemni, A. Bianco, E. Zeinstra, and A. Jimenez, "The effects of vibration during maximal graded cycling exercise: A pilot study", J. Sports Sci. Med., vol. 11, no. 3, pp. 423-429, 2012. [PMID: 24149349]

[10] B. Sperlich, H. Kleinoeder, M. De Marees, D. Quarz, J. Linville, M. Haegele, and J. Mester, "Physiological and perceptual responses of adding vibration to cycling", J. Exerc. Physiol. Online, vol. 12, no. 2, pp. 40-46, 2009.

[11] N.J. Dettori, D.C. Norvell, and D-C. Norvell, "Non-traumatic bicycle injuries : a review of the literature", Sports Med., vol. 36, no. 1, pp. 7-18, 2006. [http://dx.doi.org/10.2165/00007256-200636010-00002] [PMID: 16445308]

[12] V. Akuthota, C. Plastaras, K. Lindberg, J. Tobey, J. Press, and C. Garvan, "The effect of long-distance bicycling on ulnar and median nerves: an electrophysiologic evaluation of cyclist palsy", Am. J. Sports Med., vol. 33, no. 8, pp. 1224-1230, 2005. [http://dx.doi.org/10.1177/0363546505275131] [PMID: 16000656]

[13] M. Schwellnus, and E. Derman, "Common injuries in cycling: Prevention, diagnosis and management", S. Afr. Fam. Pract., vol. 47, no. 7, pp. 14-19, 2005.

[http://dx.doi.org/10.1080/20786204.2005.10873255]

[14] J. Srinivasan, and V. Balasubramanian, "Low back pain and muscle fatigue due to road cycling-An sEMG study", J. Bodyw. Mov. Ther., vol. 11, no. 3, pp. 260-266, 2007. [http://dx.doi.org/10.1016/j.jbmt.2006.08.009]

[15] B.D. Weiss, "Nontraumatic injuries in amateur long distance bicyclists", Am. J. Sports Med., vol. 13, no. 3, pp. 187-192, 1985. [http://dx.doi.org/10.1177/036354658501300308] [PMID: 4014534]

[16] J. Parkin, and E. Sainte Cluque, "The impact of vibration on comfort and bodily stress while cycling", In: Proceedings UTSG 46th Annual Conference, Newcastle, 2014, pp. 6-8.

[17] A. Peretti, L. Pignalosa, F. Bonomini, A. Paoli, and G. Bartolucci, "Vibrazioni su biciclette da corsa e da città", Giornale Degli Igienisti Industriali, vol. 34, no. 3, pp. 283-293, 2009.

[18] F. Giubilato, and N. Petrone, "A method for evaluating the vibrational response of racing bicycles wheels under road roughness excitation", Procedia Eng., vol. 34, pp. 409-414, 2012. [http://dx.doi.org/10.1016/j.proeng.2012.04.070]

[19] M. Olieman, R. Marin-Perianu, and M. Marin-Perianu, "Measurement of dynamic comfort in cycling using wireless acceleration sensors", Procedia Eng., vol. 34, pp. 568-573, 2012. [http://dx.doi.org/10.1016/j.proeng.2012.04.097]

[20] M. Munera, X. Chiementin, S. Murer, and W. Bertucci, "Model of the risk assessment of hand-arm system vibrations in cycling: Case of cobblestone road", Proc. Inst. Mech. Eng. P J. Sports Eng. Technol, vol. 229, pp. 1-8, 2015.

[21] J. Lépine, Y. Champoux, and J.M. Drouet, "The relative contribution of road bicycle components on vibration induced to the cyclist", Sports Eng., vol. 18, pp. 1-13, 2014.

[22] A. Doria, and L. Taraborrelli, "On the structural vibrations of bicycles: influence of materials and construction technology on the modal properties", In: Proceedings International Cycling Safety Conference, Helmond, NL, 2013, pp. 20-21.

[23] T. Tye, F.J. Gribb, and G.W. Worzala, "Modeling bicycle-rider vibrations: Implications for materials selection", MRS Bull., vol. 23, pp. 56-58, 1998. [http://dx.doi.org/10.1557/S0883769400030013]

[24] M.F. Ashby, "Materials, Bicycles, and Design", J. Metall. Mater. Trans., vol. 26, no. 12, pp. 3057-3064, 1995. [http://dx.doi.org/10.1007/BF02669435]

[25] J.C. Liu, and H.C. Wu, "Fiber direction and stacking sequence design for bicycle frame made of carbon/epoxy composite laminate", Mater. Des., vol. 31, pp. 1971-1980, 2010. [http://dx.doi.org/10.1016/j.matdes.2009.10.036] 
[26] C. Rontescu, T.D. Cicic, C.G. Amza, O. Chivu, and D. Dobrota, "Choosing the optimum material for making a bicycle frame", Metalurgija, vol. 54, no. 4, pp. 679-682, 2015.

[27] J. Vanwalleghem, Study of the damping and vibration behaviour of flax-carbon composite bicycle racing frames, M.S. thesis, Univ. of Gent, Gent, Belgique, 2010.

[28] A.K. Ray, S. Mondal, S.K. Das, and P. Ramachandrarao, "Bamboo-a functionally graded composite-correlation between microstructure and mechanical strength", J. Mater. Sci., vol. 40, no. 19, pp. 5249-5253, 2005. [http://dx.doi.org/10.1007/s10853-005-4419-9]

[29] M. Armandei, I.F. Darwish, and K. Ghavami, "Experimental study on variation of mechanical properties of a cantilever beam of bamboo", Constr. Build. Mater., vol. 101, pp. 784-790, 2015. [http://dx.doi.org/10.1016/j.conbuildmat.2015.10.078]

[30] T. Provot, X. Chiementin, E. Oudin, F. Bolaers, and S. Murer, "Validation of a high sampling rate inertial measurement unit for acceleration during running", Sensors, vol. 17, no. 9, 2017. [http://dx.doi.org/10.3390/s17091958]

[31] A.N. Thite, S. Gerguri, F. Coleman, M. Doody, and N. Fisher, "Development of an experimental methodology to evaluate the influence of a bamboo frame on the bicycle ride comfort?", Veh. Syst. Dyn., vol. 51, pp. 1287-1304, 2013. [http://dx.doi.org/10.1080/00423114.2013.797591]

[32] M.J. Griffin, Handbook of human vibration., Academic Press: London, Great Britain, 1990.

\section{C) 2017 Chiementin et al.}

This is an open access article distributed under the terms of the Creative Commons Attribution 4.0 International Public License (CC-BY 4.0), a copy of which is available at: (https:/creativecommons.org/licenses/by/4.0/legalcode). This license permits unrestricted use, distribution, and reproduction in any medium, provided the original author and source are credited. 